\title{
Weak Reciprocal Continuity and Common Fixed Point Theorems
}

\author{
Ekta H. Patel and G. M. Deheri
}

\begin{abstract}
This investigation aims to establish the importance of weak reciprocal continuity of the maps in deriving the common fixed point theorems. The existence and uniqueness of the common fixed points are obtained under generalized contractive conditions in the setting of complete metric spaces. That the rate of convergence is duly addressed is manifest in the proofs.
\end{abstract}

Index Terms-Complete metric space, fixed point, weak reciprocal continuity, compatibility.

\section{INTRODUCTION}

There are a large number of generalizations of the Banach contractive principle, popularly known as Banach fixed point theorem. This fixed point theorem was investigated under certain generalized contractions on a complete metric space. Also, the results were improved and extended in various directions, i.e., with different contraction conditions on map and with some different types of spaces. One of generalization of the Banach contraction principle finds a place in Jungck [1].

In fact, the aim of the above mentioned investigation was to represent commuting mappings as a tool for generalization. A variety of extensions, generalizations and applications of the above theorem incorporating the commuting map concept is to be found in [2], [3]. A key theorem of Meir and Keeler [4] has been developed by Park and Bae [2].

Jungck [1] has proposed a generalization of commuting mapping concept, developed properties of compatible functions and demonstrated utility of these functions in the context of metric fixed point theory by weakening the commutativity requirement.

Bisht and Joshi [5] obtained the common fixed theorems for a pair of weakly reciprocal continuous self-maps satisfying generalized contractions of Lipschitztype conditions. The problem whether there exists a contractive definition which is strong enough to generate a fixed point but does not force the map to be continuous had remained open for more than three decades. It may be observed in this context that it is known since the paper of Kannan [6], [7] that there exist maps that have discontinuity in their domain but which have fixed point. However, in all the known cases, earlier to the paper of Pant, Bisht and Arora [8], the maps involved were continuous at the fixed point. These papers generated unprecedented interest in the fixed point theory of contractive maps which in turn, resulted in intensive research on the existence of fixed points of contractive maps and the

Manuscript received November 9, 2013; revised January 27, 2014.

The authors are with the Department of Mathematics from the Sardar Patel University at Vallabh Vidhyanagar-388120, Gujarat, India (e-mail: ekta301190@gmail.com,gm.deheri@rediffmail.com). question of continuity of contractive maps at their fixed points turned into a vigorous dimension of research, which was settled in the affirmative by Pant, Bisht and Arora [8].

In the sequel, it is worthwhile to note that Pant and Bisht [9] introduced the notion of reciprocal continuity and as an application of this concept obtained the first result that established a situation in which a collection of mapping has a fixed point which is a point of discontinuity for all the mappings.

The paper of Bisht and Joshi [5] dealt with reciprocal continuity in diverse setting to establish fixed point theorems which may admit discontinuity at the fixed point. They observed that the notion of reciprocal continuity was mainly applicable to compatible mappings satisfying contractive condition. Later on, this paper became the foundation for large number of investigations [Jungck [1], Bisht and Joshi [5], Kannan [6], Kannan [7] that employed and discussed with reciprocal continuity in diverse setting to prove fixed point theorems admitting discontinuity at the fixed points.

\section{MATHEMATICAL ANALYSIS}

The method is based on the traditional iteration process and convergence there in. Due care has been taken to preserve the rate of convergence. Infact, the method adopted here is extended and modified version of some of the well known classical results on the theory of common fixed point.

To start with we prove the following:

Theorem: Let $f$ and $g$ be weakly reciprocally continuous selfmap of a Complete metric space $(X, d)$ such that

$$
\begin{gathered}
f X \subset g X \\
d^{2}\left(f_{x}, f_{y}\right) \leq \alpha d\left(f_{x,} g_{x}\right) d\left(f_{y}, g_{y}\right)+\beta d^{2}\left(f_{x}, g_{y}\right)+ \\
\gamma d\left(g_{x}, g_{y}\right) d\left(f_{y}, g_{x}\right)
\end{gathered}
$$

where $\alpha, \beta, \gamma \geq 0,0 \leq \alpha+\beta+2 \gamma<1$

If either $f$ and $g$ are compatible or $g$ compatible or $f$ compatible then $f$ and $g$ have a unique common fixed point.

Proof: Let $x_{0}$ be any point in $X$. Then, since $f X \subset g X$ there exists a sequence of points $x_{0}, x_{1}, \ldots, x_{n}, \ldots$ such that $x_{n+1}$ is the pre-image under $g$ of $f x_{n}$, that is,

$$
\begin{gathered}
f x_{0}=g x_{1}, \\
f x_{1}=g x_{2}, \\
\cdots \\
\cdots \\
f x_{n}=g x_{n+1},
\end{gathered}
$$


Also, define a sequence $\left\{y_{n}\right\}$ in $X$ by

$$
y_{n}=f x_{n}=g x_{n+1}, n=1,2, \ldots
$$

We claim that $\left\{y_{n}\right\}$ is a Cauchy sequence. Using Equation (2) one obtains the inequality

$$
\begin{gathered}
d^{2}\left(y_{n}, y_{n+1}\right) \\
=d^{2}\left(f x_{n}, f x_{n+1}\right) \\
\leq \alpha d\left(f x_{n}, g x_{n}\right) d\left(f x_{n+1}, g x_{n+1}\right)+\beta d^{2}\left(f x_{n}, g x_{n+1}\right) \\
+\gamma d\left(g x_{n}, g x_{n+1}\right) d\left(f x_{n+1}, g x_{n}\right) \\
=\alpha d\left(f x_{n}, f x_{n-1}\right) d\left(f x_{n+1}, f x_{n}\right)+\beta d^{2}\left(f x_{n}, f x_{n}\right) \\
+\gamma d\left(f x_{n-1}, f x_{n}\right) d\left(f x_{n+1}, f x_{n}\right) \\
=\alpha d\left(y_{n}, y_{n-1}\right) d\left(y_{n+1}, y_{n}\right)+\gamma d\left(y_{n-1}, y_{n}\right) d\left(y_{n+1}, y_{n-1}\right) \\
\leq \alpha d\left(y_{n}, y_{n-1}\right) d\left(y_{n+1}, y_{n}\right)+\gamma d\left(y_{n-1}, y_{n}\right) d\left(y_{n+1}, y_{n}\right) \\
+\gamma d\left(y_{n-1}, y_{n}\right) d\left(y_{n}, y_{n-1}\right) \\
\leq \alpha\left[\frac{d^{2}\left(y_{n}, y_{n-1}\right)}{2}+\frac{d^{2}\left(y_{n+1}, y_{n}\right)}{2}\right] \\
{\left[\begin{array}{l}
2 \\
+\gamma\left[\frac{d^{2}\left(y_{n}, y_{n-1}\right)}{2}+\frac{d^{2}\left(y_{n}, y_{n+1}\right)}{2}+2 \gamma \frac{d^{2}\left(y_{n}, y_{n-1}\right)}{2}\right]
\end{array}\right.}
\end{gathered}
$$

which leads to

$$
\begin{aligned}
& 2 d^{2}\left(y_{n}, y_{n+1}\right) \leq \alpha\left[d^{2}\left(y_{n}, y_{n-1}\right)+d^{2}\left(y_{n+1}, y_{n}\right)\right] \\
&+\gamma\left[d^{2}\left(y_{n}, y_{n-1}\right)+d^{2}\left(y_{n+1}, y_{n}\right)\right] \\
&+3 \gamma d^{2}\left(y_{n}, y_{n-1}\right) \\
&(2-\alpha-\gamma) d^{2}\left(y_{n}, y_{n+1}\right) \leq(\alpha+3 \gamma) d^{2}\left(y_{n}, y_{n-1}\right)
\end{aligned}
$$

Thus

$$
d^{2}\left(y_{n}, y_{n+1}\right) \leq\left(\frac{\alpha+3 \gamma}{2-\alpha-\gamma}\right) d^{2}\left(y_{n}, y_{n-1}\right)
$$

where

$$
\frac{(\alpha+3 \gamma)}{(2-\alpha-\gamma)}<1
$$

Let

$$
\lambda=\left(\frac{\alpha+3 \gamma}{2-\alpha-\gamma}\right)^{1 / 2},
$$

Then $0<\lambda<1$.

Therefore, we conclude that

$$
\begin{aligned}
d\left(y_{n}, y_{n+1}\right) & \leq \lambda d\left(y_{n}, y_{n-1}\right) \\
& \leq \lambda^{2} d\left(y_{n-1}, y_{n-2}\right) \\
& \cdots \\
& \cdots \\
& \cdots \\
& \leq \lambda^{n} d\left(y_{1}, y_{0}\right)
\end{aligned}
$$

Consequently, $d\left(y_{n}, y_{n+1}\right) \rightarrow 0$ as $n \rightarrow \infty$

Therefore, $\left\{y_{n}\right\}$ is a Cauchy sequence. Since $X$ is complete, there exists a point in $X$ such $y_{n} \rightarrow t$ as $n \rightarrow \infty$. Moreover,

$$
y_{n}=f x_{n}=g x_{n+1} \rightarrow t
$$

Suppose that $f$ and $g$ are compatible mappings. Now, weak reciprocal continuity of $f$ and $g$ implies that

$$
f g x_{n} \rightarrow f t \text { or } g f x_{n} \rightarrow g t
$$

Let $g f x_{n} \rightarrow g t$. Then compatibility of $f$ and $g$ yields

$$
\lim _{n \rightarrow \infty} d\left(f g x_{n}, g f x_{n}\right)=0,
$$

and so $f g x_{n} \rightarrow g t$.

By virtue of Equation (3) this also yields

$$
g f x_{n}=g g x_{n+1} \rightarrow g t
$$

Using equation (2) one gets,

$$
\begin{gathered}
d^{2}\left(f t, f g x_{n}\right) \\
\leq \alpha d(f t, g t) d\left(f g x_{n}, g g x_{n}\right)+\beta d^{2}\left(f t, g g x_{n}\right)+ \\
\gamma d\left(g t, g g x_{n}\right) d\left(f g x_{n}, g t\right)
\end{gathered}
$$

This leads to,

$$
\begin{aligned}
d^{2}(f t, g t) \leq & \alpha d(f t, g t) d(g t, g t)+\beta d^{2}(f t, g t) \\
& +\gamma d(g t, g t) d(g t, g t)
\end{aligned}
$$

which gives

$$
d^{2}(f t, g t) \leq \beta d^{2}(f t, g t)
$$

so $f t=g t$, since $\beta<1$.

Again compatibility of $\mathrm{f}$ and $g$ implies commutativity at coincidence points.

Hence

$$
f g t=g f t \text { and } g f t=f g t=f f t
$$

Once again, using Equation (2) one finds that

$$
\begin{gathered}
d^{2}(f t, f f t) \\
\leq \alpha d(f t, g t) d(f f t, g f t)+\beta d^{2}(f t, g f t) \\
+\gamma d(g t, g f t) d(f f t, g t)
\end{gathered}
$$

This means

$$
\begin{aligned}
d^{2}(f t, f f t) \leq & \alpha d(f t, f t) d(f f t, f f t)+\beta d^{2}(f t, f f t) \\
& +\gamma d(f t, f f t) d(f f t, f t)
\end{aligned}
$$

Therefore,

$$
d^{2}(f t, f f t) \leq(\beta+\gamma) d^{2}(f t, f f t)
$$

So $f t=f f t$, since $(\beta+\gamma)<1$

Hence, $f t=f f t=g f t$ and $f t$ is a common fixed point of $f$ and $g$.

Next suppose that $f g x_{n} \rightarrow f t$.Then

$$
f X \subseteq g X \Rightarrow f t=g u \text { for some } u \in X
$$

and $f g x_{n} \rightarrow g u$.

Compatibility of $f$ and $g$ implies, $g f x_{n} \rightarrow g u$. By virtue of Equation (3) this yields 


$$
g g x_{n+1}=g f x_{n} \rightarrow g u \text {. }
$$

Using Equation (2) one gets,

$$
\begin{gathered}
d^{2}\left(f u, f g x_{n}\right) \\
\leq \alpha d(f u, g u) d\left(f g x_{n}, g g x_{n}\right)+\beta d^{2}\left(f u, g g x_{n}\right) \\
+\gamma d\left(g u, g g x_{n}\right) d\left(f g x_{n}, g u\right)
\end{gathered}
$$

Consequently,

$$
\begin{aligned}
d^{2}(f u, g u) \leq & \alpha d(f u, g u) d(g u, g u)+\beta d^{2}(f u, g u) \\
& +\gamma d(g u, g u) d(g u, g u)
\end{aligned}
$$

which yields

$$
d^{2}(f u, g u) \leq \beta d^{2}(f u, g u)
$$

Therefore, $f u=g u$, as $\beta<1$.

Compatibility of $f$ and $g$ implies that $f g u=g f u$ and hence,

$$
g g u=g f u=f g u=f f u .
$$

Finally, using Equation (2) one obtains the inequality

$$
\begin{gathered}
d^{2}(f u, f f u) \\
\leq \alpha d(f u, g u) d(f f u, g f u)+\beta d^{2}(f u, g f u) \\
+\gamma d(g u, g f u) d(f f u, g u)
\end{gathered}
$$

This provides,

$$
\begin{gathered}
d^{2}(f u, f f u) \\
\leq \alpha d(f u, f u) d(f f u, f f u)+\beta d^{2}(f u, f f u) \\
+\gamma d(f u, f f u) d(f f u, f u)
\end{gathered}
$$

Thus,

$$
d^{2}(f u, f f u) \leq(\beta+\gamma) d^{2}(f u, f f u)
$$

And so, $f u=f f u$, since $\beta+\gamma<1$.

Hence $f u=f f u=g f u$ and $f u$ is a common fixed point of $f$ and $g$.

Now, suppose that $f$ and $g$ are $g$ - compatible. Then weak reciprocal continuity of $f$ and $g$ implies that

$$
f g x_{n} \rightarrow f t \text { or } g f x_{n} \rightarrow g t
$$

Let $g f x_{n} \rightarrow g t$. Then $g$ - compatibility of $f$ and $g$ yields

$$
\begin{gathered}
\lim _{n \rightarrow \infty} d\left(f f x_{n}, g f x_{n}\right)=0 \\
\Rightarrow f f x_{n} \rightarrow g t .
\end{gathered}
$$

Using Equation (2).one gets

$$
\begin{gathered}
d^{2}\left(f t, f f x_{n}\right) \\
\leq \alpha d(f t, g t) d\left(f f x_{n}, g f x_{n}\right)+\beta d^{2}\left(f t, g f x_{n}\right) \\
+\gamma d\left(g t, g f x_{n}\right) d\left(f f x_{n}, g t\right)
\end{gathered}
$$

This result in,

$$
\begin{aligned}
d^{2}(f t, g t) \leq & \alpha d(f t, g t) d(g t, g t)+\beta d^{2}(f t, g t) \\
& +\gamma d(g t, g t) d(g t, g t)
\end{aligned}
$$

which gives

$$
d^{2}(f t, g t) \leq \beta d^{2}(f t, g t)
$$

so $=g t$, since $\beta<1$

Since $g$ - compatibility implies commutativity at coincidence points, one concludes that

$$
f g t=g f t
$$

and

$$
g g t=g f t=f g t=f f t .
$$

Using Equation (2) one arrives at

$$
\begin{gathered}
d^{2}(f t, f f t) \leq \\
\alpha d(f t, g t) d(f f t, g f t)+\beta d^{2}(f t, g f t)+ \\
\gamma d(g t, g f t) d(f f t, g t)
\end{gathered}
$$

This suggests that

$$
\begin{aligned}
d^{2}(f t, f f t) \leq & \alpha d(f t, f t) d(f f t, f f t)+\beta d^{2}(f t, f f t) \\
& +\gamma d(f t, f f t) d(f f t, f t)
\end{aligned}
$$

which means

$$
d^{2}(f t, f f t) \leq(\beta+\gamma) d^{2}(f t, f f t)
$$

so, $f t=f f t$, since $\beta+\gamma<1$.

Hence,

$$
f t=f f t=g f t
$$

and $f t$ is a common fixed point of $f$ and $g$.

Next suppose that $f g x_{n} \rightarrow f t$. Then

$$
f X \subseteq g X \Rightarrow f t=g u \text { for some } u \in X
$$

and

$$
f g x_{n} \rightarrow g u
$$

$g$-compatibility of $f$ and $g$ implies, $g f x_{n} \rightarrow g u$. By virtue of Equation (3) this yields

$$
f f x_{n} \rightarrow g u
$$

Using Equation (2) one derives

$$
\begin{gathered}
d^{2}\left(f u, f f x_{n}\right) \\
\leq \alpha d(f u, g u) d\left(f f x_{n}, g f x_{n}\right)+\beta d^{2}\left(f u, g f x_{n}\right)+ \\
\gamma d\left(g u, g f x_{n}\right) d\left(f f x_{n}, g u\right)
\end{gathered}
$$

which yields 


$$
\begin{aligned}
d^{2}(f u, g u) & \leq \alpha d(f u, g u) d(g u, g u)+\beta d^{2}(f u, g u) \\
& +\gamma d(g u, g u) d(g u, g u)
\end{aligned}
$$

leading to

$$
d^{2}(f u, g u) \leq \beta d^{2}(f u, g u)
$$

and so $f u=g u$, since $\beta<1$.

Again $g$ - compatibility implies commutativity at coincidence points. Thus,

$$
f g u=g f u
$$

and hence,

$$
g g u=g f u=f g u=f f u .
$$

Using Equation (2) one finds that

$$
\begin{gathered}
d^{2}(f u, f f u) \leq \alpha d(f u, g u) d(f f u, g f u)+ \\
\beta d^{2}(f u, g f u)+\gamma d(g u, g f u) d(f f u, g u)
\end{gathered}
$$

which results in

$$
\begin{aligned}
d^{2}(f u, f f u) \leq & \alpha d(f u, f u) d(f f u, f f u)+\beta d^{2}(f u, f f u) \\
& +\gamma d(f u, f f u) d(f f u, f u)
\end{aligned}
$$

leading to

$$
d^{2}(f u, f f u) \leq(\beta+\gamma) d^{2}(f u, f f u)
$$

This says that,

$$
f u=f f u, \text { since } \beta+\gamma<1 .
$$

Hence,

$$
f u=f f u=g f u
$$

and

$f u$ is a common fixed point of $f$ and $g$.

Finally, suppose that $f$ and $g$ are $f$ - compatible. Now, weak reciprocal continuity of $f$ and g implies that

$$
f g x_{n} \rightarrow f t \text { or } g f x_{n} \rightarrow g t .
$$

Let $g f x_{n} \rightarrow g t$. Then $f$-compatibility of $f$ and $g$ and in view of $g f x_{n}=g g x_{n+1}$, one conclude that

$$
\lim _{n \rightarrow \infty} d\left(f g x_{n}, g g x_{n}\right)=0
$$

Thus,

$$
f g x_{n} \rightarrow g t
$$

Using Equation (2) one arrives at

$$
\begin{gathered}
d^{2}\left(f t, f g x_{n}\right) \\
\leq \alpha d(f t, g t) d\left(f g x_{n}, g g x_{n}\right)+\beta d^{2}\left(f t, g g x_{n}\right)+ \\
\gamma d\left(g t, g g x_{n}\right) d\left(f g x_{n}, g t\right)
\end{gathered}
$$

So,

which gives

$$
\begin{gathered}
d^{2}(f t, g t) \leq \alpha d(f t, g t) d(g t, g t)+\beta d^{2}(f t, g t) \\
+\gamma d(g t, g t) d(g t, g t)
\end{gathered}
$$

$$
d^{2}(f t, g t) \leq \beta d^{2}(f t, g t)
$$

Hence,

$$
f t=g t, \text { since } \beta<1 .
$$

Since $f$ - compatibility implies commutativity at coincidence points, we have

$$
f g t=g f t \text { and } g g t=g f t=f g t=f f t .
$$

Using Equation (2) one derives

$$
\begin{gathered}
d^{2}(f t, f f t) \leq \\
\alpha d(f t, g t) d(f f t, g f t)+\beta d^{2}(f t, g f t)+ \\
\gamma d(g t, g f t) d(f f t, g t)
\end{gathered}
$$

leading to

$$
\begin{gathered}
d^{2}(f t, f f t) \\
\leq \alpha d(f t, f t) d(f f t, f f t)+\beta d^{2}(f t, f f t) \\
+\gamma d(f t, f f t) d(f f t, f t)
\end{gathered}
$$

resulting in

$$
d^{2}(f t, f f t) \leq(\beta+\gamma) d^{2}(f t, f f t)
$$

Therefore, $f t=f f t$, since $\beta+\gamma<1$.

Hence $f t=f f t=g f t$ and $\mathrm{ft}$ is a comman fixed point of $f$ and $g$.

Next suppose that $f g x_{n} \rightarrow f t$.

Then $f X \subseteq g X$ implies that $f t=g u$ for some $u \in$ $X$ and $f g x_{n} \rightarrow g u$.

$f$-compatibility of $f$ and $g$ implies, $g g x_{n} \rightarrow g u$.

Using Equation (2), one finds that

$$
\begin{gathered}
d^{2}\left(f u, f g x_{n}\right) \\
\leq \alpha d(f u, g u) d\left(f g x_{n}, g g x_{n}\right)+\beta d^{2}\left(f u, g g x_{n}\right)+ \\
\gamma d\left(g u, g g x_{n}\right) d\left(f g x_{n}, g u\right)
\end{gathered}
$$

which gives

$$
\begin{aligned}
d^{2}(f u, g u) \leq & \alpha d(f u, g u) d(g u, g u)+\beta d^{2}(f u, g u) \\
& +\gamma d(g u, g u) d(g u, g u)
\end{aligned}
$$

This leads to

$$
d^{2}(f u, g u) \leq \beta d^{2}(f u, g u)
$$

so $=g u$, since $\beta<1$.

Again $f$ - compatibility of $f$ and $g$ yields

$$
f g u=g f u
$$

and hence, 


$$
g g u=g f u=f g u=f f u .
$$

Using (2) One conclude that

$$
\begin{gathered}
d^{2}(f u, f f u) \leq \alpha d(f u, g u) d(f f u, g f u)+ \\
\beta d^{2}(f u, g f u)+\gamma d(g u, g f u) d(f f u, g u)
\end{gathered}
$$

which means

$$
\begin{aligned}
d^{2}(f u, f f u) \leq & \alpha d(f u, f u) d(f f u, f f u)+\beta d^{2}(f u, f f u) \\
& +\gamma d(f u, f f u) d(f f u, f u)
\end{aligned}
$$

resulting in

$$
d^{2}(f u, f f u) \leq(\beta+\gamma) d^{2}(f u, f f u)
$$

That is, $f u=f f u$, since $\beta+\gamma<1$.

Hence, $f u=f f u=g f u$ and $f u$ is a common fixed point of $f$ and $g$.

For uniqueness of common fixed point, let $t_{1}, t_{2}$ be two distinct points such that

$$
f t_{1}=t_{1}=g t_{1}, \text { and } f t_{2}=t_{2}=g t_{2} .
$$

Then, from Equation (2) it follows that

$$
\begin{gathered}
d^{2}\left(t_{1}, t_{2}\right) \\
=d^{2}\left(f t_{1}, f t_{2}\right) \\
\leq \alpha d\left(f t_{1}, g t_{1}\right) d\left(f t_{2}, g t_{2}\right)+\beta d^{2}\left(f t_{1}, g t_{2}\right) \\
+\gamma d\left(g t_{1}, g t_{2}\right) d\left(f t_{2}, g t_{1}\right) \\
=\alpha d\left(t_{1}, t_{1}\right) d\left(t_{2}, t_{2}\right)+\beta d^{2}\left(t_{1}, t_{2}\right)+\gamma d\left(t_{1}, t_{2}\right) d\left(t_{2}, t_{1}\right) \\
=(\beta+\gamma) d^{2}\left(t_{1}, t_{2}\right)
\end{gathered}
$$

which is a contradiction as $(\beta+\gamma)<1$.

Hence, $t_{1}=t_{2}$. That means, $f$ and $g$ have a unique common fixed point in $X$.

Theorem: Let $f, g$ and $h$ be weakly reciprocally continuous self maps of a complete metric space $(X, d)$ such that

$$
\begin{gathered}
f X \subset h X \subset g X \\
d^{2}\left(f_{x}, f_{y}\right) \leq \alpha d\left(f_{x}, g_{x}\right) d\left(f_{y}, g_{y}\right)+\beta d^{2}\left(g_{x}, h_{x}\right)+ \\
\gamma d\left(f_{x}, h_{x}\right) d\left(f_{y}, h_{y}\right)
\end{gathered}
$$

where $\alpha, \beta, \gamma \geq 0,0 \leq \alpha+\gamma<1$

If $f, g$ and $h$ are compatible then $f, g$ and $h$ have a unique common fixed point.

Proof: Let $x_{0}$ be any point in $X$. Then, since $f X \subset h X \subset g X$, there exists a sequence of points $x_{0}, \ldots, x_{n}, \ldots$ such that $x_{n+1}$ is the pre-image under $g$ and $h$ of $f x_{n}$, that is,

$$
\begin{gathered}
f x_{0}=g x_{1} \text { and } f x_{0}=h x_{1}, \\
f x_{1}=g x_{2} \text { and } f x_{1}=h x_{2}, \\
\ldots \\
\cdots \\
\ldots \\
f x_{n}=g x_{n+1} \text { and } f x_{n}=h x_{n+1}, \\
\ldots \\
\ldots
\end{gathered}
$$

Also, define a sequence $\left\{y_{n}\right\}$ in $X$ by

$$
y_{n}=f x_{n}=g x_{n+1}=h x_{n+1}, n=1,2, \ldots
$$

We claim that $\left\{y_{n}\right\}$ is a Cauchy sequence. Using Equation (33) one obtains the inequality

$$
\begin{gathered}
d^{2}\left(y_{n}, y_{n+1}\right) \\
=d^{2}\left(f x_{n}, f x_{n+1}\right) \\
\leq \alpha d\left(f x_{n}, g x_{n}\right) d\left(f x_{n+1}, g x_{n+1}\right)+\beta d^{2}\left(g x_{n}, h x_{n}\right) \\
+\gamma d\left(f x_{n}, h x_{n}\right) d\left(f x_{n+1}, h x_{n+1}\right) \\
=\alpha d\left(f x_{n}, f x_{n-1}\right) d\left(f x_{n+1}, f x_{n}\right)+\beta d^{2}\left(f x_{n-1}, f x_{n-1}\right) \\
+\gamma d\left(f x_{n}, f x_{n-1}\right) d\left(f x_{n+1}, f x_{n}\right) \\
=\alpha d\left(y_{n}, y_{n-1}\right) d\left(y_{n+1}, y_{n}\right)+\gamma d\left(y_{n}, y_{n-1}\right) d\left(y_{n+1}, y_{n}\right) \\
\leq \alpha\left[\frac{d^{2}\left(y_{n}, y_{n-1}\right)}{2}+\frac{d^{2}\left(y_{n+1}, y_{n}\right)}{2}\right] \\
+\gamma\left[\frac{d^{2}\left(y_{n}, y_{n-1}\right)}{2}+\frac{d^{2}\left(y_{n}, y_{n+1}\right)}{2}\right]
\end{gathered}
$$

which leads to

$$
\begin{gathered}
2 d^{2}\left(y_{n}, y_{n+1}\right) \leq \alpha\left[d^{2}\left(y_{n}, y_{n-1}\right)+d^{2}\left(y_{n+1}, y_{n}\right)\right] \\
+\gamma\left[d^{2}\left(y_{n}, y_{n-1}\right)+d^{2}\left(y_{n}, y_{n+1}\right)\right] \\
(2-\alpha-\gamma) d^{2}\left(y_{n}, y_{n+1}\right) \leq(\alpha+\gamma) d^{2}\left(y_{n}, y_{n-1}\right)
\end{gathered}
$$

Thus,

$$
d^{2}\left(y_{n}, y_{n+1}\right) \leq\left(\frac{\alpha+\gamma}{2-\alpha-\gamma}\right) d^{2}\left(y_{n}, y_{n-1}\right)
$$

where,

$$
\frac{(\alpha+\gamma)}{(2-\alpha-\gamma)}<1
$$

Let

$$
\lambda=\left(\frac{\alpha+\gamma}{2-\alpha-\gamma}\right)^{1 / 2}
$$

Then $0<\lambda<1$.

Therefore, we conclude that

$$
\begin{aligned}
d\left(y_{n}, y_{n+1}\right) & \leq \lambda d\left(y_{n}, y_{n-1}\right) \\
& \leq \lambda^{2} d\left(y_{n-1}, y_{n-2}\right) \\
& \cdots \\
& \cdots \\
& \cdots \\
\leq & \lambda^{n} d\left(y_{1}, y_{0}\right)
\end{aligned}
$$

Consequently, $d\left(y_{n}, y_{n+1}\right) \rightarrow 0$ as $n \rightarrow \infty$

Therefore, $\left\{y_{n}\right\}$ is a Cauchy sequence. Since $X$ is complete, there exists a point in $X$ such that $y_{n} \rightarrow t$ as $n \rightarrow \infty$. Moreover,

$$
y_{n}=f x_{n}=g x_{n+1}=h x_{n+1} \rightarrow t .
$$


Suppose that $f$ and $g$ are compatible mappings. Now, weak reciprocal continuity of $f$ and $g$ implies that

$$
f g x_{n} \rightarrow f t \text { or } g f x_{n} \rightarrow g t .
$$

Also, $g$ and $h$ are compatible mappings. Now, weak reciprocal continuity of $g$ and $h$ implies that

$$
g h x_{n} \rightarrow g t \text { or } h g x_{n} \rightarrow h t .
$$

As well as $h$ and $f$ are compatible mappings. Now, weak reciprocal continuity of $h$ and $f$ implies that

$$
f h x_{n} \rightarrow f t \text { or } h f x_{n} \rightarrow h t .
$$

Let $\quad g f x_{n} \rightarrow g t, g h x_{n} \rightarrow g t$ and $h f x_{n} \rightarrow$. Then compatibility of $f$ and $g$ leads to

$$
\lim _{n \rightarrow \infty} d\left(f g x_{n}, g f x_{n}\right)=0,
$$

So, $f g x_{n} \rightarrow g t$.

Now, compatibility of $g$ and gives

$$
\lim _{n \rightarrow \infty} d\left(g h x_{n}, h g x_{n}\right)=0
$$

And so, $h g x_{n} \rightarrow g t$.

Again compatibility of $f$ and $h$ turns in

Thus, $f h x_{n} \rightarrow h t$.

$$
\lim _{n \rightarrow \infty} d\left(f h x_{n}, h f x_{n}\right)=0
$$

By virtue of Equation (34) this also yields

$$
h h x_{n+1}=h f x_{n} \rightarrow h t \text { and } g t=\lim _{n \rightarrow \infty}\left(h g x_{n}\right)=h t .
$$

Using Equation (33) one gets,

$$
\begin{aligned}
& d^{2}\left(f t, f h x_{n}\right) \leq \alpha d(f t, g t) d\left(f h x_{n}, g h x_{n}\right)+ \\
& \beta d^{2}(g t, h t)+\gamma d(f t, h t) d\left(f h x_{n}, h h x_{n}\right)
\end{aligned}
$$

This leads to

$$
\begin{gathered}
d^{2}(f t, h t) \leq \alpha d(f t, g t) d(h t, g t)+\beta d^{2}(g t, h t) \\
+\gamma d(f t, h t) d(h t, h t)
\end{gathered}
$$

Therefore,

$$
d^{2}(f t, h t) \leq 0
$$

So we must have

$$
f t=h t=g t
$$

But then compatibility of $\mathrm{f}$ and $g$ implies commutativity at coincidence points.

Hence,

$$
f f t=f g t=g f t
$$

Again compatibility of $\mathrm{g}$ and $h$ implies commutativity at coincidence points.

Hence,

$$
g f t=g h t=h g t
$$

Once again compatibility of $\mathrm{f}$ and $h$ implies commutativity at coincidence points.

Hence,

$$
h g t=h f t=f h t
$$

Now, using Equation (33) one finds that

$$
\begin{gathered}
d^{2}(f t, f f t) \\
\leq \alpha d(f t, g t) d(f f t, g f t)+\beta d^{2}(g t, h t)+ \\
\gamma d(f t, h t) d(f f t, h f t)
\end{gathered}
$$

This means

$$
\begin{aligned}
d^{2}(f t, f f t) \leq & \alpha d(f t, f t) d(f f t, f f t)+\beta d^{2}(f t, f t) \\
& +\gamma d(f t, f t) d(f f t, f f t)
\end{aligned}
$$

Therefore,

$$
d^{2}(f t, f f t) \leq 0
$$

So we must have

$$
f t=f f t=g f t=h f t
$$

And $f t$ is a common fixed point of $f, g$ and $h$. Next suppose that $f g x_{n} \rightarrow f t, f h x_{n} \rightarrow f t$ and $h g x_{n} \rightarrow h t$. then

$f X \subseteq g X \Rightarrow f t=g u$ for some $u \in X$ and $f g x_{n} \rightarrow g u$.

Also $f X \subseteq h X \Rightarrow f t=h u$ for some $u \in X$ and $f h x_{n} \rightarrow h u$.

Clearly $h X \subseteq g X \Rightarrow h t=g u$ for some $u \in X$ and $h g x_{n} \rightarrow g u$.

Compatibility of $f$ and $g$ implies, $g f x_{n} \rightarrow g u$. By virtue of Equation (34) this yields

$$
g g x_{n+1}=g f x_{n} \rightarrow g u
$$

Again compatibility of $h$ and $g$ implies, $g h x_{n} \rightarrow g u$. By virtue of Equation (34) this results in

$$
h h x_{n+1}=h g x_{n+1} \rightarrow g u .
$$

since $f t=g u$ and $h u=f t$.

Hence,

$$
g u=f t=h u .
$$

Using Equation (33) one gets,

$$
\begin{aligned}
& d^{2}\left(f u, f h x_{n}\right) \leq \alpha d(f u, g u) d\left(f h x_{n}, g h x_{n}\right)+ \\
& \beta d^{2}(g u, h u)+\gamma d(f u, h u) d\left(f h x_{n}, h h x_{n}\right)
\end{aligned}
$$

Consequently,

$$
\begin{aligned}
d^{2}(f u, h u) \leq & \alpha d(f u, g u) d(h u, g u)+\beta d^{2}(g u, h u) \\
& +\gamma d(f u, h u) d(h u, g u)
\end{aligned}
$$

which gives 


$$
d^{2}(f u, h u) \leq 0
$$

So we must have

$$
f u=h u=g u .
$$

Compatibility of $f$ and $g$ implies that $f g u=g f u$ and hence,

$$
g g u=g f u=f g u=f f u .
$$

Again compatibility of $f$ and $h$ implies that $h f u=f h u$ and hence,

$$
f f u=f h u=h f u .
$$

Finally, using Equation (33) one obtains the inequality

$$
\begin{aligned}
& d^{2}(f u, f f u) \leq \alpha d(f u, g u) d(f f u, g f u)+ \\
& \beta d^{2}(g u, h u)+\gamma d(f u, h u) d(f f u, h f u)
\end{aligned}
$$

This provides

$$
\begin{aligned}
d^{2}(f u, f f u) \leq & \alpha d(f u, f u) d(f f u, f f u)+\beta d^{2}(f u, f u) \\
& +\gamma d(f u, f u) d(f f u, f f u) \\
& d^{2}(f u, f f u) \leq 0
\end{aligned}
$$

So we conclude that

$$
f u=f f u=g f u=h f u .
$$

Therefore, $f u$ is a common fixed point of $f, g$ and $h$.

For uniqueness of common fixed point, let $t_{1}, t_{2}$ be two distinct points such that

$$
f t_{1}=t_{1}=g t_{1}=h t_{1} \text {, and } f t_{2}=t_{2}=g t_{2}=h t_{2} \text {. }
$$

Then, from Equation (33) it follows that

$$
\begin{gathered}
d^{2}\left(t_{1}, t_{2}\right) \\
=d^{2}\left(f t_{1}, f t_{2}\right) \\
\leq \alpha d\left(f t_{1}, g t_{1}\right) d\left(f t_{2}, g t_{2}\right)+\beta d^{2}\left(g t_{1}, h t_{1}\right) \\
+\gamma d\left(f t_{1}, h t_{1}\right) d\left(f t_{2}, h t_{2}\right) \\
=\alpha d\left(t_{1}, t_{1}\right) d\left(t_{2}, t_{2}\right)+\beta d^{2}\left(t_{1}, t_{1}\right)+\gamma d\left(t_{1}, t_{1}\right) d\left(t_{2}, t_{2}\right)
\end{gathered}
$$

That is,

$$
d^{2}\left(t_{1}, t_{2}\right) \leq 0
$$

$$
t_{1}=t_{2}
$$

That means $f, g$ and $h$ have a unique common fixed point in $X$.

\section{CONCLUSION}

Although our results are more sharp and strengthened, the rate of convergence is a little bit slow. Probably, this may be due to the fact that the condition on the maps and the space are not that strong to increase the rate of convergence. Here we observe that our results are obtained in the most generalized settings.

\section{REFERENCES}

[1] G. Jungck, "Compatible mappings and common fixed points," Journal of Mathematics and Mathematical Sciences, vol. 9, pp. 771-779, 1986.

[2] S. Park and Bae, "Jong Sook extensions of a fixed point theorem of Meir and Keeler," Arikib Mathematicae, vol. 19, pp. 223-228, 1981.

[3] F. Brain, "Mapping with a common," Mathematical Seminar Notes, vol. 7, pp. 81-83, 1979.

[4] A. Meir and E. Keeler, "A theorem on contraction mappings," Journal of Mathematical Analysis and Applications, vol. 2, pp. 526-529, 1969.

[5] R. K. Bisht, and R. U. Joshi, "Common fixed point theorems of weakly reciprocal continuous maps," Journal of the Indian Mathematical Society, vol. 79, pp. 1-12, no. 1-4, 2012.

[6] R. Kannan, "Some results on fixed points," Bulletin of Calcutta Mathematical Society, vol. 60, pp. 71-76, 1968.

[7] R. Kannan, "Some results on fixed point-II," American Mathematical Monthly, vol. 76, pp. 405-408, 1969.

[8] R. P. Pant, R. K. Bisht, and D. Arora, "Weak reciprocal continuity and fixed point theorems," Ann University Ferrara, vol. 57, pp. 181-190, 2011.

[9] R. P. Pant and R. K. Bisht, Common fixed point theorems under a new continuity condition, Ann University, Ferrara, vol. 58, pp. 127-141, 2012.

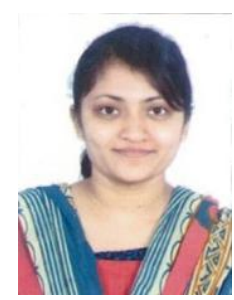

Ekta H. Patel was born on $30^{\text {th }}$ November 1990, at Wanakbori Thermal Power Station, G.S.E.C.L Colony, Type-3, 52/3, Distict-Kheda, Gujarat, India.

After the completion of master of science with first class distinction in mathematics in April 2013 from Sardar Patel University, Vallabh Vidyanagar. She has been pursuing master of philosophy program from July 2013 at the same University in the post graduate Department of Mathematics. She has participated in personal workshop.

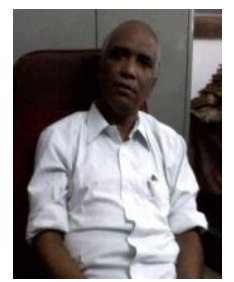

Gunamant Deheri received his $\mathrm{PhD}$ degree from IIT Kanpur. He is working as a reader in Mathematics Department of Mathematics at Sardar Patel University, Vallabh Vidyanagar.

$\mathrm{He}$ has received Hari Ohm Ashram Award four times and also Balkanjibari Science Trust Award. His area of research is Tribology. He has published more than 150 papers in International and National Journals.

So we must have 